\title{
VENOUS THROMBOSIS IN THE MEDICAL UNIT- WHAT IS DIFFERENT?
}

\author{
Vidya Sanjay Nagar1, Rahul Kadu², Mayur Hedau³, Basavaraj Sajjan', Rudrarpan Chatterjee ${ }^{5}$
}

${ }^{1}$ Associate Professor, Department of Medicine, Grant Government Medical College and Sir JJ Hospital, Mumbai. ${ }^{2}$ Assistant Professor, Department of Medicine, Grant Government Medical College and Sir JJ Hospital, Mumbai. 3Assistant Professor, Department of Medicine, Grant Government Medical College and Sir JJ Hospital, Mumbai. 4 Junior Resident, Department of Medicine, Grant Government Medical College and Sir JJ Hospital, Mumbai. 5Junior Resident, Department of Medicine, Grant Government Medical College and Sir JJ Hospital, Mumbai.

ABSTRACT
BACKGROUND
Deep venous thrombosis is an important comorbidity in admitted patients with a reported incidence of up to 17.46 cases per
100000 patients. Though seen typically as a surgical complication, it is often encountered in the medically ill.

\section{MATERIALS AND METHODS}

A prospective observational study was carried out in a tertiary care centre. Patients admitted in the medical ward without any surgical illness or comorbidity were screened using the Well's clinical criteria. Development of venous thrombosis was confirmed using Doppler ultrasound and CT pulmonary angiography. Statistical analysis was carried out using descriptive statistics at a confidence level of $95 \%$ with a p value of less than 0.05 considered significant.

\section{RESULTS}

Median age at presentation was 48 years with a male to female ratio of 1.5:1. Prevalence of deep venous thrombosis was $29.4 \%$ and that of pulmonary thromboembolism $3.92 \%$. A high probability of venous thrombosis predicted by the Well's scoring system was associated with subsequent development of deep venous thrombosis and pulmonary embolism. 17 out of 74 asymptomatic patients were found to have venous thrombosis which was statistically significant. 4 patients had proximal DVT, 10 had distal and 16 had both proximal and distal involvement. Duration of immobilisation was found to be the most important risk factor with a median duration of 12 days in those who developed venous thrombosis. Right ventricular hypokinesia on 2D Echo and CT pulmonary angiography were found to be diagnostic for venous thrombosis. 4 out of 30 patients of DVT and 1 out of 4 patients of pulmonary thromboembolism died.

\section{CONCLUSION}

Deep venous thrombosis has a high prevalence in the medical ward. The Well's clinical criteria is a good tool to predict deep venous thrombosis and pulmonary thromboembolism. Multiple acute medical conditions predispose to the development of venous thrombosis with prolonged immobilisation being the most important independent risk factor. Development of venous thrombosis during admission is associated with a poor outcome.

\section{KEYWORDS}

Deep Venous Thrombosis, Pulmonary Thromboembolism, Well's Criteria.

HOW TO CITE THIS ARTICLE: Nagar VS, Kadu R, Hedau M, et al. Venous thrombosis in the medical unit- What is different? J. Evolution Med. Dent. Sci. 2017;6(39):3128-3130, DOI: 10.14260/Jemds/2017/674

\section{BACKGROUND}

Rudolf Virchow, in his seminal paper on thrombosis, delineated the triad of factors, which in due sequence predispose to thrombosis. These were, namely, stasis, endothelial injury and hypercoagulability of blood.

Venous thrombosis, comprising of deep venous thrombosis and pulmonary thromboembolism constitute a significant cause of morbidity and mortality that is potentially preventable.

A study from India put the incidence of venous thrombosis at 17.46 per 100000 patients.[1] $64 \%$ of these cases were deemed non-surgical cases.[1]

Financial or Other, Competing Interest: None.

Submission 02-04-2017, Peer Review 04-05-2017,

Acceptance 10-05-2017, Published 15-05-2017.

Corresponding Author:

Dr. Rudrarpan Chatterjee,

Room 409, 300 Resident Doctor's Hostel,

JJ Hospital, Byculla, Mumbai-400008.

E-mail: rudi.gmc2009@gmail.com

DOI: $10.14260 /$ jemds $/ 2017 / 674$
Hospitalisation for acute medical illness is estimated to have an 8-fold higher risk of developing deep venous thrombosis.[2] Pulmonary thromboembolism, with $75 \%$ of cases accounted for from the medical ward, is an important cause of sudden death in hospitalised patients. [3] There is a strong association between venous thrombosis and the chances of developing subsequent venous thromboembolism.

Deep venous thrombosis is typically seen as an illness and complication of the surgically ill. A number of medical illnesses also predispose a patient to conditions optimal for the development of deep venous thrombosis.

The Well's clinical probability scores are a set of criteria that determine pre-test probability of venous thrombosis.[4],[5] A good clinical scoring system to predict venous thrombosis, will lead to earlier institution of prophylaxis for venous thrombosis and prevent potential morbidity and mortality.

\section{Objectives}

To evaluate the utility Well's clinical scoring system for predicting deep venous thrombosis in the medical unit and to 
elucidate the risk factors for venous thrombosis in the medical unit.

\section{MATERIALS AND METHODS}

This was a prospective observational study at a tertiary care centre. 102 patients admitted in the ward with a primarily medical illness were screened for deep venous thrombosis. Well's score was used to assess pre-test probability of venous thrombosis in all patients, and patients were followed up during their hospital stay for development of deep venous thrombosis either when they were symptomatic for the same or prior to planned discharge if asymptomatic. Any patient with a history of surgical illness or comorbidity in the last 12 weeks and those with a history of thromboprophylaxis were excluded from the study. Sample size was estimated from an estimated true proportion of $23 \%$ from literature at a precision of 0.1 at a confidence level of $95 \%$. Shapiro-Wilk test was used to test normality. Duration of immobilisation was compared between those with and without DVT using the Mann-Whitney U test. Pearson's chi square test and Fisher's exact test were used to test relationship between categorised independent and dependent variables. Stata SE 13.1 was the statistical software used for analysis.

\section{RESULTS}

A total of 102 patients were screened who met the inclusion and exclusion criteria. Median age was 48 years with a male to female ratio of 1.5:1.

DVT was present in 30 cases (29.4\%) and absent in 72. 4 out of 102 cases had pulmonary thromboembolism (3.92\%). There was a significant association with increasing age more than 60 and the development of DVT $(\mathrm{p}<0.001)$ though no such significance was present with pulmonary thromboembolism. There was no significant association between sex and development of DVT.

All patients were screened and assigned a probability of high, moderate or low chance of DVT and PE on admission as per the Well's clinical criteria. DVT was diagnosed in 28 out of 44, 2 out of 38 and 0 out of 20 patients assigned categories of high, moderate and low probability respectively. This was very highly significant $(43.831, \mathrm{p}<0.001)$ on statistical analysis. Similarly 3 out of 14,1 out of 20 and 0 out of 68 patients had pulmonary thromboembolism in the categories of high, moderate and low risk respectively as per the Well's clinical criteria. $(\mathrm{p}<0.001)$

28 cases were symptomatic for venous thrombosis at the time of Doppler examination and 74 were not. 13 out of the 28 and 17 out of 74 cases were found to have DVT on Doppler examination. This was found to be statistically significant (5.383, $\mathrm{p}=0.02)$. Therefore, absence of symptoms does not rule out the presence of DVT. 4 patients had proximal DVT, 10 had distal involvement and 16 had both proximal as well as distal involvement. Pulmonary thromboembolism was present in 2 out of the 4 cases of proximal DVT and none of the cases of distal DVT. 2 of the 16 patients with both proximal and distal involvement went on to develop PE. There was no statistical significance to this. $(\mathrm{p}=0.065)$.

Further, our study elucidated the risk factors for venous thrombosis and embolism in medically ill patients (figure 1). Of all the risk factors, duration of immobilisation was found to be the most important factor. On further analysis, the median duration was 5 days for patients who did not develop venous thrombosis as compared to 12 days for those who did. (figure 2). This was found to be very highly statistically significant on the Mann-Whitney U test. $(\mathrm{p}<0.0001)$.

Deep venous thrombosis was found in many common causes of hospitalisation in the medical ward. (Table 1.) Diabetes mellitus was the only medical comorbidity that was significantly associated with venous thrombosis in this study. $(\mathrm{p}=0.008)$.

On imaging acute right ventricular hypokinesia on 2D echo was found to be diagnostic for pulmonary thromboembolism as confirmed by CT angiography $(\mathrm{p}=0.015)$.

DVT was found to have significant association with mortality, though the same was not established for pulmonary thromboembolism in our study. Out of 30 cases of deep venous thrombosis, 26 were discharged and 4 died. $(\mathrm{p}=0.006)$ In the group of patients with pulmonary thromboembolism, out of 4 patients, 3 were discharged and 1 died.

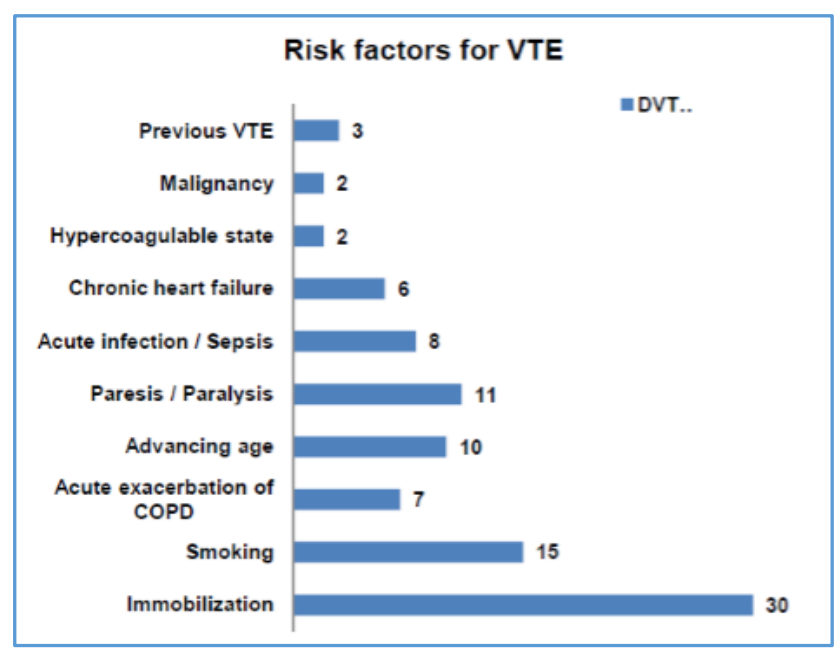

Figure 1. Risk Factors for Venous Thrombosis in our Study.

VTE venous thromboembolism. COPD- chronic obstructive pulmonary disease

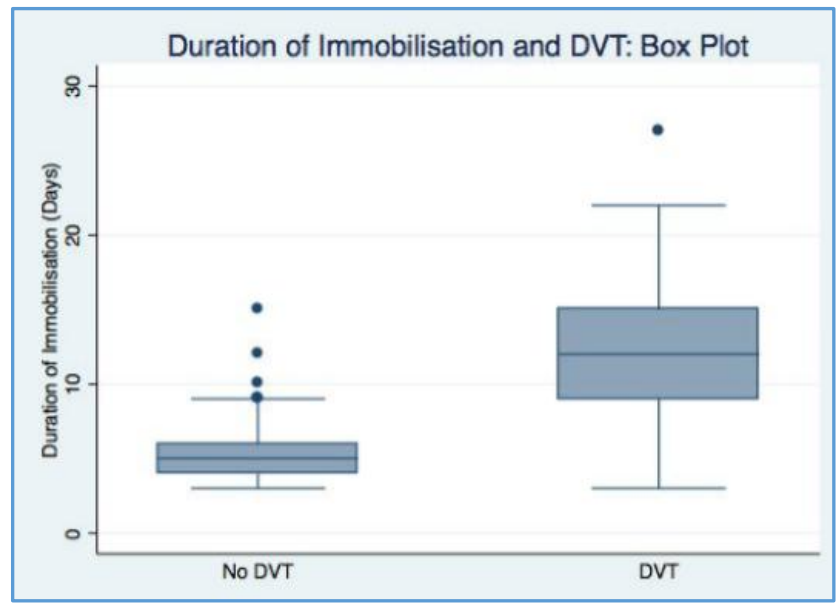

Figure 2. Box Plot Showing Significantly Higher Duration of Immobilisation in the Group with DVT 


\begin{tabular}{|c|c|c|c|c|}
\hline \multirow{2}{*}{ Disease } & \multicolumn{2}{|c|}{ DVT } & \multirow{2}{*}{ Total } & \multirow{2}{*}{ P-Value } \\
\hline & Present & Absent & & \\
\hline Stroke & $9(42.9)$ & 12 (57.1) & $21(100)$ & 0.129 \\
\hline Sepsis & $(44.4)$ & $10(55.6)$ & & \\
\hline $\begin{array}{l}\text { COPD with acute } \\
\text { exacerbation }\end{array}$ & $7(41.2)$ & $10(58.8)$ & $17(100)$ & 0.244 \\
\hline HIV & $6(50)$ & $6(50)$ & $12(100)$ & 0.173 \\
\hline IHD & $6(24)$ & $19(76)$ & $25(10$ & 0.494 \\
\hline & $(75)$ & $2(25)$ & & 008 \\
\hline Renal fail & $3(37.5)$ & $5(62.5)$ & & 0.690 \\
\hline Maligna & $1(100)$ & $0(0)$ & & \\
\hline $\begin{array}{l}\text { Hypercoagulable } \\
\text { state }\end{array}$ & 2 (66.7) & $1(33.3)$ & $3(100)$ & 0.206 \\
\hline \multicolumn{5}{|c|}{$\begin{array}{l}\text { Table 1. Distribution of Cases According to Disease. Note: } \\
\text { Figures in Parentheses Denote Percentages. } \\
\text { COPD-Chronic obstructive pulmonary disease, HIV- Human } \\
\text { immunodeficiency virus, IHD-Ischaemic heart disease, } \\
\text { DM-Diabetes mellitus }\end{array}$} \\
\hline
\end{tabular}

\section{DISCUSSION}

Venous thrombosis has been traditionally thought of as a comorbidity of surgical illness and therapy, chiefly due to its association with immobilisation. The myth that venous thrombosis is less in Asian populations as compared to western ones is now debunked.[6] In the last decade, several Indian studies have demonstrated the incidence of deep venous thrombosis comparable to western literature in the Indian population. In a recent study, $75 \%$ of patients admitted in the medical ward or intensive care unit were found to be at high risk of developing deep venous thrombosis. [7] Our study found deep venous thrombosis in $29.4 \%$ of patients.

The Well's clinical probability scoring system for deep venous thrombosis and pulmonary thromboembolism were proposed in 1997 and are a simple screening tool for assessing risk of developing venous thrombosis.[4], [5] As demonstrated in this study, Well's score is an accurate tool in predicting the development of deep venous thrombosis. Adequate prophylaxis needs to be initiated in the form of mechanical compression stockings or anticoagulation for such patients.

Right ventricular hypokinesia on 2D echo and CT pulmonary angiography were both found to be excellent tools in diagnosing DVT in those with a high pre-test probability as already described in literature.[8] Asymptomatic DVT was present in $23 \%$ of patients in our study. This puts greater emphasis on clinical suspicion on the basis of the Well's scoring system.

Prolonged immobilisation is one of the most important independent risk factors for DVT.[9] While relevant in all surgical cases, in medicine prolonged immobilisation is particularly important in neurological conditions. Of note is the fact that all the cases of DVT that developed in patients of chronic obstructive pulmonary disease had prolonged periods of immobilisation due to ventilator dependence, both invasive and non-invasive, in the intensive care unit.

A high number of patients with stroke, spinal cord disease, congestive cardiac failure, diabetes mellitus, ischaemic heart disease, acute exacerbation of COPD, malignancy and sepsis had deep venous thrombosis in this study. All of these acute medical conditions are strong independent risk factors for VTE.

To cement these findings with a study of higher precision would require a larger sample size. This is a limitation of this study. Being a prospective study, a much longer followup period would be required to enrol the sample size required, preferably across multiple centres to achieve a higher precision.

Deep venous thrombosis is a predictor of poor outcome in hospitalised patients as per literature and was reinforced by the findings of this study.

\section{CONCLUSION}

Though deep venous thrombosis is considered a surgical complication, it is very common in the medicine ward as it represents a significant comorbidity that affects outcomes adversely. The Well's clinical criteria are still an excellent tool to screen for deep venous thrombosis and pulmonary thromboembolism in the ward. Many acute medical illnesses are associated with venous thrombosis with the common risk factor being prolonged immobilisation.

\section{REFERENCES}

[1] Lee AD, Stephen E, Agarwal S, et al. Venous thromboembolism in India. Eur J Vasc Endovasc Surg 2009;37(4):482-5.

[2] Bonthier J. The venous thrombotic risk in non-surgical patients. Drugs 1996;52(Suppl 7):16-29.

[3] Cohen AT, Alikhan R, Arcelus JI, et al. Assessment of venous thromboembolism risk and the benefits of thromboprophylaxis in medical patients. Thromb Haemost 2005;94(4):750-9.

[4] Wells PS, Anderson DR, Bormanis J, et al. Value of assessment of pre-test probability of deep-vein thrombosis in clinical management. Lancet 1997;350(9094):1795-8.

[5] Wells PS, Anderson DR, Rodger M, et al. Derivation of a simple clinical model to categorize patients' probability of pulmonary embolism: increasing the models utility with the Implored D-dimer. Thromb Haemost 2000;83(3):416-20.

[6] Bhan S, Dhaon BK, Gulati Y, et al. DVT prophylaxis: a multicentric study. Indian J Orphtop 2004;38(3): 178-82.

[7] Pandey A, Patni N, Singh M, et al. Assessment of risk and prophylaxis for DVT and PE in medically ill patients during their early days of hospital stay at a tertiary care centre in a developing country. Vasc Health Risk Manag 2009;5:643-8.

[8] Vieillard-Baron A, Page B, Augarde R, et al. Acute cor pulmonale in massive pulmonary embolism: incidence, echocardiographic pattern, clinical implications and recovery rate. Intensive Care Med 2001;27(9):1481-6.

[9] Wells PS, Owen C, Doucette S, et al. Does this patient have deep vein thrombosis? JAMA 2006;295(2):199. 207. 